\title{
Efeitos Genéticos e de Ambiente sobre Produção de Leite e Intervalo de Partos em Rebanhos Leiteiros Monitorados por Sistema de Informação*
}

\author{
Ana Rosa Zambianchi ${ }^{3}$, Maria Armênia Ramalho de Freitas ${ }^{1,3}$, Carmem Silva Pereira ${ }^{2,3}$
}

\begin{abstract}
RESUMO - Este estudo foi realizado para analisar a influência de alguns fatores de meio sobre a produção de leite e o intervalo de partos e estimar os parâmetros genéticos da produção de leite aos 305 dias (P305) e do intervalo de partos (IP). Foram analisadas 3044 lactações de 1568 vacas da raça Holandesa, paridas no período de 1988 a 1995, pertencentes a rebanhos monitorados pelo Sistema de Informação DAISY (The Dairy Information System). Nas análises dos fatores não-genéticos, foi utilizado o método dos quadrados mínimos e para estimação dos parâmetros genéticos, o método da máxima verossimilhança restrita (REML), utilizando-se análises uni e bivariadas.

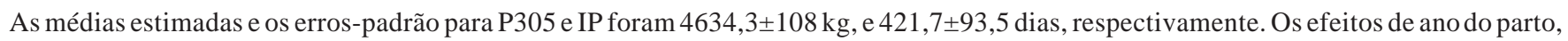
rebanho ou nível de produção e idade da vaca em classes influenciaram todas as características estudadas. As estimativas de herdabilidade para P305 e IEP variaram de 0,24 a 0,43 e 0,06 a 0,07, respectivamente. As correlações genética, fenotípica e de ambiente entre produção de leite até 305 dias e intervalo de partos foram 0,42;0,06; e 0,11, respectivamente. Houve variação genética aditiva para produção de leite e correlação genética positiva, porém indesejável, entre as características analisadas, sugerindo que a seleção para a alta produção de leite poderia acarretar aumento no intervalo de parto.
\end{abstract}

Palavras-chave: fertilidade, produção de leite, sistema DAISY, vacas Holandesa

\section{Genetic and Environmental Aspects on Milk Yield and Calving Interval of Dairy Herds Monitored by Computerized Information Systems}

\begin{abstract}
This study was carried out to analyze the influence of some environmental effects on the milk yield and calving interval and to estimate the genetic parameters at 305-day milk yield (P305), and calving interval (IP). Data from 3044 lactations of 1568 Holstein cows, calved from 1988 to 1995 belonging to dairy herds monitored by DAISY (The Dairy Information System), were used. The Least Squares Method was used to study the environment effects and the Derivative-Free Restricted Maximum Likelihood (REML) algorithm was used to estimate genetic parameters using univariate and bivariate sire model. The estimated means and standard errors were $4634.4 \pm 108 \mathrm{~kg}$ and $421.7 \pm$ 93.5 days for P 305 and IP, respectively. Calving year, herd or production level and age of cow affected all studied traits. The estimated heritabilities ranged from 0.24 to .43 for P305 and .06 to .07 for IP. The genetic, phenotypic and environmental correlation between 305 -day milk yield and calving interval were $.42, .12$ and .11, respectively. There was an additive genetic variation for milk yield and a positive genetic correlation, but undesirable relationship among the traits, sugesting that the selection for high milk yield could increase the calving interval
\end{abstract}

Key Words: DAISY system, fertility, Holstein cows, milk production

\section{Introdução}

A produtividade da pecuária leiteira brasileira, quando comparada à dos países desenvolvidos, deixa muito a desejar, visto que nossos rebanhos apresentam baixa eficiência reprodutiva e produtiva, reflexo, entre outras causas, da não-utilização das tecnologias já disponíveis para o setor leiteiro. Um dos entraves neste processo é a falta de informações disponíveis; entretanto, nos últimos anos, tem-se esforçado para aumentar o número de animais controlados nos rebanhos e padronizar a coleta dos dados zootécnicos, elementos indispensáveis para correta avaliação das características de importância econômica, aumentando a acurácia do valor genético dos animais e melhorando a eficácia dos programas de seleção. Neste contexto, o uso dos sistemas computadorizados de informação (SI) constitui-se em instrumento importante no monitoramento dos rebanhos leiteiros, pois permite o controle detalhado dos mesmos e o fluxo contínuo das informações de modo preciso e rápido. Sua importância já foi bem descrita por TOMASZEWSKI (1993) e GUILHERMINO (1994). Sabe-se que a eficiência econômica de qualquer

\footnotetext{
* Apoio CNPq/FAPESP.

${ }^{1}$ EEZRP/Instituto de Zootecnia/CPA/SAA-SP, C.P. 206, Ribeirão Preto CEP.14030-670.

2 Departamento de Zootecnia - Escola de Veterinária da UFMG.

${ }^{3}$ Bolsista: CNPq e CAPES.
} 
sistema de produção depende do bom desempenho dos animais e dos fatores genéticos e de meio que interferem na expressão das características de importância econômica (SPAIN et al., 1994; WIGGANS et al., 1994). FREITAS et al. (1996), no Brasil, analisando aspectos da produção de rebanhos leiteiros comerciais monitorados por sistema computacional de informação, observaram melhora nos índices de desempenho para a maior parte das características analisadas e concluíram que os SI propiciam condições para que as decisões do produtor sejam tomadas com maior precisão, melhorando a produtividade, contribuindo para reduzir custos.

A raça Holandesa é uma das principais raças responsáveis pela produção de leite no país, utilizada não somente como raça pura, como também intensamente em cruzamentos com outros grupos raciais. Em razão da melhora nas condições oferecidas aos rebanhos desta raça, a média de produção de leite tem aumentado consideravelmente nos últimos anos (FREITAS, 1981; RORATO, 1988; e MATOS, 1996). Consta da literatura que a média dos intervalos de partos de vacas desta raça se situa entre 394 e 438 dias. É conhecido que vacas que parem todos os anos produzem mais leite por dia de vida útil (FREITAS et al., 1985). Com relação ao estudo dos parâmetros genéticos e de ambiente para as características em estudo, atualmente, a literatura brasileira tem mostrado vários resultados para a raça Holandesa, entre os quais se destacam os de RIBAS et al. (1993), SILVA (1995), RICHTER et al. (1995), MATOS et al. (1996) e PINHEIRO (1996). As estimativas de herdabilidade do intervalo de partos encontradas na literatura brasileira, em geral, confirmam as expectativas de valores reduzidos para esta característica, com raras exceções (TEIXEIRA et al., 1994; SILVA, 1995). Assim, este estudo foi conduzido para analisar a influência de alguns fatores não-genéticos sobre a produção de leite e o intervalo de partos e estimar os parâmetros genéticos (herdabilidade e correlações), em rebanhos da raça Holandesa, monitorados por sistema de informação.

\section{Materiais e Métodos}

Neste estudo, foram utilizados 3044 lactações e registros do desempenho reprodutivo de 1568 vacas da raça Holandesa no total de 1038 intervalos de partos, em 15 rebanhos monitorados pelo sistema computacional de informação DAISY (The Dairy Information System), em projeto desenvolvido pelo Instituto de Zootecnia/ SAA-São Paulo. Nas análises preliminares para estu- do dos fatores de meio (ano do parto, idade da vaca, rebanho ou nível de produção e estação do parto), o efeito da idade da vaca ao parto foi analisado como classes. As classes foram as seguintes:

1) animais com idade ao parto menor ou igual a 36 meses; 2) animais com idade ao parto entre $37 \mathrm{e}$ 48 meses; 3) animais com idade ao parto entre $49 \mathrm{e}$ 60 meses; 4) animais com idade ao parto entre $61 \mathrm{e}$ 72 meses; 5) animais com idade ao parto entre $73 \mathrm{e}$ 84 meses; 6) animais com idade ao parto entre $85 \mathrm{e}$ 92 meses; e 7) animais com idade ao parto maior que 92 meses.

Foi utilizado o programa LSMLMW, descrito por HARVEY (1990), com o seguinte modelo fixo:

$\mathrm{Y}_{\mathrm{ijkl}}=\mu+\mathrm{R}_{\mathrm{i}}+\mathrm{I}_{\mathrm{j}}+\mathrm{A}_{\mathrm{k}}+\mathrm{E}_{1}+\mathrm{e}_{\mathrm{ijkl}}$

em que $\mathrm{Y}_{\mathrm{ijkl}}=$ variável dependente; $\mu=$ média geral; $\mathrm{R}_{\mathrm{i}}=$ efeito do rebanho $(\mathrm{i}=1, \ldots, 15) ; \mathrm{I}_{\mathrm{j}}=$ efeito da idade ao vaca ao parto em classes $(\mathrm{j}=1,2, \ldots, 7)$; $\mathrm{A}_{\mathrm{k}}=$ efeito do ano do parto $(\mathrm{k}=1, \ldots 7) ; \mathrm{E}_{1}=$ efeito da estação do parto, águas $=1$ (outubro a março) e seca $=2$ (abril a setembro) $(1=1,2)$; e $\mathrm{e}_{\mathrm{ijkl}}=$ erro aleatório associado a cada observação ijkl $\sim \mathrm{N}\left(0, \mathrm{I}_{\mathrm{e}}{ }^{2}\right)$.

Para estimar os componentes de variância das características em estudo, foi empregado o método da Máxima Verossimilhança Restrita (REML), sob um modelo de touro, por intermédio do software MTDFREML (Multiple Trait Derivative-Free Restricted Maximum Likelihood), descrito por BOLDMAN et al. (1993), sob o seguinte modelo misto na forma matricial:

$$
\mathbf{Y}=\mathbf{X} \boldsymbol{\beta}+\mathbf{Z v}+\mathbf{e}
$$

em que $\mathrm{Y}=$ vetor de informações; $\beta=$ vetor de efeitos fixos; $X=$ matriz de incidência que associa $\beta$ com $X ; \mathrm{u}=$ vetor dos efeitos aleatórios [genético aditivo]; $Z=$ matriz de incidência que associa elementos de u com Y; e = vetor dos efeitos aleatórios residuais; e

Pressuposições: $\mathrm{V}(\mathrm{v})=\mathrm{G}=\mathrm{A} \sigma_{\mathrm{r}}^{2} ; \mathrm{V}(\mathrm{e})=\mathrm{R}=\mathrm{I}$ $\sigma^{2}{ }_{\mathrm{e}} ; \operatorname{Cov}\left(v, \mathrm{e}^{\prime}\right)=0$

Os efeitos significativos no estudo preliminar foram considerados fixos - ano do parto, rebanho e nível de produção e idade da vaca em classes. Com o objetivo de melhorar a conectabilidade e diminuir o número de subclasses, devido à pequena quantidade de dados por rebanho para estudo de parâmetros genéticos, os dados foram agrupados em três níveis de produção (NP1, NP2 e NP3). Para estabelecimento dos mesmos, utilizou-se, como produção média, o intervalo compreendido entre a média geral mais ou menos meio desvio-padrão da produção de leite. As produções abaixo e acima deste intervalo 
Rev. bras. zootec.

foram consideradas baixas e altas, respectivamente. Assim, utilizou-se um modelo em que se incluía rebanho entre os efeitos fixos e um outro em que o efeito de rebanho foi substituído por nível de produção. Foram eliminados do arquivo geral touros com menos de três filhas. Foi realizada uma análise bicaráter, para obtenção dos componentes de (co)variância das características intervalo de partos e produção total de leite, sob um modelo de touro.

\section{Resultados e Discussão}

A média estimada para produção de leite foi de $4634,3 \pm 108,7 \mathrm{~kg}$, valor semelhante aos encontrados em outros trabalhos brasileiros, com a mesma raça, já citados anteriormente. As médias estimadas de produção de leite até os 305 dias de acordo com rebanho, oscilaram entre o mínimo de $2577 \pm 203 \mathrm{~kg}$ e o máximo de $8417 \pm 234 \mathrm{~kg}$ evidenciando as diferenças de manejo e potencial genético dentro do grupo de rebanhos analisados. Os resultados da análise de variância da produção de leite, observados na Tabela 1, demonstram que os efeitos de rebanho, ano do parto e idade da vaca em classes foram altamente significativos $(\mathrm{P}<0,01)$, concordando com vários autores (RIVEROS MEDINA, 1979; REIS, 1983; CONCEIÇÃO JR., 1991; e RICHTER et al., 1995). As médias estimadas da produção de leite, segundo a idade da vaca em classes, mostraram aumento da produção, com o avançar da idade, e declínio a partir das classes 4 e 5, que correspondia à quarta lactação. Este resultado confirma outros citados por COSTA et al. (1982). O fator estação do parto não influiu na produção de leite dos rebanhos analisados. Os produtores envolvidos neste programa fazem, em geral, boa suplementação de alimentos dos mesmos durante todo ano.

Tabela 1 - Análise de variância da produção de leite Table 1 - Analysis of variance of milk yield

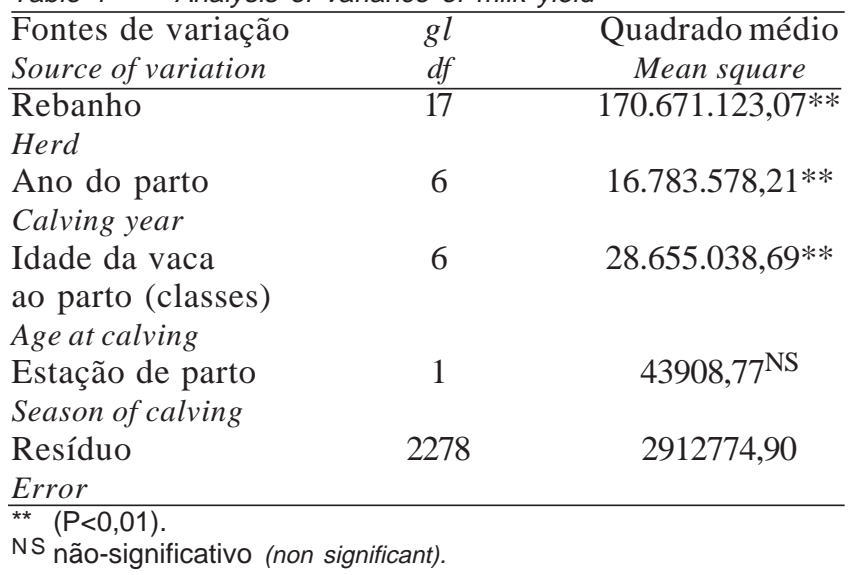

$\mathrm{Na}$ análise do intervalo de partos, a média estimada e o erro-padrão foram, respectivamente, 422 (13,8 meses) e 94 dias. Estes valores, em relação aos encontrados na literatura brasileira para a raça Holandesa, estão próximos do ideal preconizado para o melhoramento da eficiência reprodutiva dos rebanhos leiteiros. A Tabela 2 mostra a análise de variância dos IEPs, na qual se constata que, entre os efeitos fixos, o ano do parto foi altamente significativo. Sabese que o desempenho reprodutivo da vaca é geralmente medido pelo intervalo de partos e seus componentes, período de gestação e período de serviço. Como o primeiro é mais ou menos constante, as variações do intervalo de partos decorrem predominantemente das mudanças no período de serviço, que, por sua vez, sofre a influência de fatores que estão na dependência da taxa de detecção de cio - período do parto ao primeiro serviço, taxas de concepção e intervalo de serviços. O efeito da idade da vaca ao parto em classes sobre esta característica também foi altamente significativo. Verificou-se pequeno aumento no IEP, com o avanço da idade, concordando com a literatura (SIQUEIRA, 1976; MANSO et al., 1980; e COSTA, 1980). Este fato é explicado, em alguns casos, pelo retardamento da involução uterina em certos animais e pelo restabelecimento do ciclo estral, que se tornam mais demorados com o avançar da idade. Os efeitos de nível de produção e estação do parto não foram significativos sobre o intervalo de partos. Houve decréscimo nas médias estimadas segundo o ano do parto para o intervalo de partos. A redução nestes valores, ao longo dos anos em estudo, pode ser atribuída à melhora na taxa de detecção de cio nos rebanhos, como também a reflexos de melhor alimentação e de manejo mais adequados.

Tabela 2 - Análise de variância do intervalo de partos Table 2 - Analysis of variance of calving interval

\begin{tabular}{lcc}
\hline $\begin{array}{l}\text { Fontes de variação } \\
\text { Source of variation }\end{array}$ & $\begin{array}{c}g l \\
d f\end{array}$ & $\begin{array}{c}\text { Quadrado médio } \\
\text { Mean square }\end{array}$ \\
\hline $\begin{array}{l}\text { Ano do parto } \\
\text { Calving year }\end{array}$ & 6 & $189409,18^{* * *}$ \\
$\begin{array}{l}\text { Estação de parto } \\
\text { Season of calving } \\
\text { Nível de produção } \\
\text { Level of production }\end{array}$ & 1 & $5382,14^{\mathrm{NS}}$ \\
$\begin{array}{l}\text { Idade da vaca } \\
\text { ao parto (classes) }\end{array}$ & 2 & $4209,22^{\mathrm{NS}}$ \\
$\begin{array}{l}\text { Age at calving } \\
\text { Resíduo }\end{array}$ & 5 & $48856,71^{* *}$ \\
Error & & \\
$* *$ (P<0,05). & 1023 & 8739,66 \\
$\mathrm{NS}$ não-significativo (non significant).
\end{tabular}


Com relação às estimativas dos parâmetros genéticos da produção de leite, cumpridas as restrições impostas pela metodologia descritas anteriormente, visando à melhor conectabilidade dos dados, o arquivo de análises foi reduzido a 856 lactações. A média e o desvio-padrão da produção de leite neste caso foram, respectivamente, $5197 \pm 1870 \mathrm{~kg}$. É importante salientar que a razão principal para a eliminação de informações foi o número excessivo de touros usados pelos produtores de leite, com menos de três filhas. Isto ocorre, na maioria dos casos, devido à falta de planejamento dos acasalamentos que, nas propriedades leiteiras, é feito sem objetivo definido, prática bastante comum no país. Isto demonstra o pouco conhecimento dos critérios de escolha de reprodutores para uso em seus rebanhos, por parte dos produtores de leite, dificultando, assim, a avaliação genética dos reprodutores em uso no nosso país.

As estimativas de herdabilidade obtidas para essa característica diferiram conforme o modelo de análise (Tabela 3). Os resultados são semelhantes aos valores de herdabilidade para produção de leite, encontrados para rebanhos da raça Holandesa em vários estudos nas condições brasileiras, e seguem a mesma tendência internacional, que aponta valores pouco mais elevados, quando resultantes de análises com a metodologia da máxima verossimilhança restrita. Sob análise bicaráter, estes valores aumentaram para 0,36 , no modelo em que se incluiu o efeito de rebanho, e 0,43, quando se usou nível de produção. Estes valores, juntamente com as correlações genética $\left(r_{\mathrm{a}}\right)$, ambiental $\left(r_{\mathrm{e}}\right)$ e fenotípica $\left(r_{\mathrm{p}}\right)$ entre estas características, encontram-se na Tabela 4.

A estimativa de herdabilidade do intervalo de

Tabela 3 - Coeficiente de herdabilidade da produção de leite até 305 dias $\left(\mathrm{h}^{2}\right)$, com componentes de variância genética aditiva $\left(\sigma_{a}{ }^{2}\right)$ e residual $\left(\sigma_{e}{ }^{2}\right)$ considerando nível de produção ou rebanho no modelo

Table 3 - Heritability estimated of 305-day milk yield $\left(h^{2}\right)$ and components of genetic $\left(\sigma^{2}{ }_{a}\right)$ and residual $\left(\sigma^{2}{ }_{e}\right)$ variance considering production level or herd in the model

\begin{tabular}{lccc}
\hline $\begin{array}{l}\text { Modelo } \\
\text { Model }\end{array}$ & $\mathrm{h}^{2}$ & $\sigma^{2}{ }_{\mathrm{a}}$ & $\sigma_{\mathrm{e}}^{2}$ \\
\hline $\begin{array}{l}\text { Rebanho } \\
\begin{array}{l}\text { Herd } \\
\text { Nível de produção } \\
\text { Level of production }\end{array}\end{array}$ & 0,24 & $485.980,47$ & 1526424,27 \\
\hline
\end{tabular}

partos, a partir de 540 lactações obtidas pela análise univariada, quando se considerou no modelo o efeito fixo de rebanho, foi de 0,07 e 0,09, quando foi considerado nível de produção. Estes resultados confirmam os mencionados por SILVA (1995), no Brasil, que relatam valores reduzidos para herdabilidade do intervalo de partos, em estudos com a mesma raça, evidenciando ser esta característica dependente fundamentalmente dos fatores de meio que interferem em sua expressão. Em geral, a literatura menciona que vacas de alto potencial genético para produção de leite tendem a apresentar problemas reprodutivos, quando comparadas às de menor nível de produção, o que não se observou neste estudo. Este fato mostra que, em termos reprodutivos, os rebanhos vêm sendo razoavelmente controlados, pois as características reprodutivas sofrem grande influência dos efeitos de meio facilmente detectados pelo sistema de informação. VAN ARENDONK et al. (1989) destacam que a reduzida fertilidade nestes animais não está diretamente relacionada ao aumento na produção de leite, mais sim na perda de peso e na condição corporal das vacas, e que a magnitude do impacto negativo na produção de leite ou reprodução vai depender do balanço de energia no início da lactação. Esses autores citam ainda que, nestes casos, a eficiência reprodutiva não é comprometida, se forem oferecidas aos animais energia e proteína em níveis adequados, para suportar altos níveis de produção de leite no início da lactação. Com referência aos valores encontrados neste estudo para as correlações genéticas entre produção de leite e intervalo de partos 0,42 e 0,36 , pode-se inferir que a seleção para produção de leite pode acarretar aumento no intervalo de partos.
Tabela 4 - Coeficientes de herdabilidade da produção de leite até 305 dias ( $\left.\mathrm{h}^{2} \mathrm{P} 305\right)$ e do intervalo de partos $\left(h^{2}\right.$ IEP), e correlações genética $\left(r_{a}\right)$, ambiental $\left(r_{e}\right)$ e fenotípica $\left(r_{p}\right)$ entre produçẫo de leite e intervalo de partos

Table 4 - Heritability of 305-day milk yield $\left(h^{2}{ }_{P 305}\right)$ and calving interval $\left(h^{2}{ }_{\text {IEP }}\right)$ and genetic $\left(r_{a}\right)$, environmental $\left(r_{e}\right)$ and phenotipic $\left(r_{p}\right)$ correlations between milk yield and calving interval

\begin{tabular}{lccccc}
\hline $\begin{array}{l}\text { Modelo } \\
\text { Model }\end{array}$ & $\mathrm{h}^{2}{ }_{\mathrm{P} 305}$ & $\mathrm{~h}^{2}$ IEP & $r_{\mathrm{a}}$ & $r_{\mathrm{e}}$ & $r_{\mathrm{p}}$ \\
$\begin{array}{l}\text { Rebanho } \\
\begin{array}{l}\text { Herd } \\
\text { Nível de produção } \\
\text { Level production }\end{array}\end{array}$ & 0,36 & 0,07 & 0,42 & 0,11 & 0,06 \\
\hline
\end{tabular}




\section{Conclusões}

As herdabilidades da produção de leite obtidas neste seguem a mesma tendência dos outros países, que demonstram ser possível, pela seleção, a obtenção de aumento na produção de leite. Com relação ao IEP, os valores de herdabilidade confirmam ser esta característica praticamente dependente dos fatores de meio. A correlação genética entre as características em estudo sugere relação positiva, porém indesejável, entre produção de leite e intervalo de partos.

\section{Referências Bibliográficas}

BOLDMAN, K.G., KRIESE, L.A., VAN VLECK, L.D. et al. 1993. A manual for use for MTDFREML. A set of programs to obtain estimates of variance and covariances [DRAFT]. Lincoln, Department of Agriculture. Agricultural Research Service. 120 p.

CONCEIÇÃO JR., V. Aspectos de ambiente e genéticos das produções de leite e gordura em vacas da raça Holandesa. Belo Horizonte UFMG: Escola Veterinária, 1991. 194p. Dissertação (Mestrado em Zootecnia) - Universidade Federal de Minas Gerais, 1991.

COSTA, C.N. Fatores genéticos e de meio na produção de leite e eficiência reprodutiva de um rebanho Holandês. Viçosa: UFV, 1980. 93p. Dissertação (Mestrado em Zootecnia) Universidade Federal de Viçosa, 1980.

COSTA, C.N., MILAGRES, J.C., GARCIA, J.A. et al. 1982. Fatores genéticos e de meio na eficiência reprodutiva de um rebanho Holandês. R. Soc. Bras. Zootec., 11(1):86-102.

FREITAS, M.A.R. Aspectos fenotípicos e genéticos da produção de leite e suas relações com a reprodução em um rebanho da raça Holandesa. Ribeirão Preto: USP, 1981. 146p. Tese (Doutorado em Genética) - Universidade de São Paulo, 1981.

FREITAS, M.A.R., LÔBO, R.B., CARDOSO, V.L. 1985. Características reprodutivas como causa de variação na produção de leite. Bol. Ind. Anim., 42(1):131-140.

FREITAS, M.A.R., ZAMBIANCHI, A.R. Utilização de sistema de informação no monitoramento de rebanhos leiteiros. I Controle leiteiro em rebanhos comuns. In: REUNIÃO ANUAL DA SOCIEDADE BRASILEIRA DE MELHORAMENTO ANIMAL, 1, 1996. Ribeirão Preto. Anais... Ribeirão Preto: USP, 1996. p.128.

GUILHERMINO, M.M. 1994. The use of information systems by dairy farmers in England and Wales. England: University of Reading. 261p.

HARVEY, W.R. 1990. User's guide for LSMLMW and MIXMDL. PC-2 Version (Mixed Model Least-Squares and Maximum Likelihood Computer Program). Columbus: Ohio. 91p.

MANSO, H.C., PRIMO, G.B., CAMPELlO, E.C.B. et al. Aspectos da eficiência reprodutiva de um rebanho Holandês, P.O., explorado na Zona da Mata do Estado de Pernambuco. In: REUNIÃO ANUAL DA SOCIEDADE BRASILEIRA DE ZOOTECNIA, 27, 1980, Fortaleza. Anais... Fortaleza: SBZ, p.219, 1980.

MATOS, R.S. Parâmetros genéticos para produção de leite e gordura da raça Holandesa no Estado do Rio Grande do Sul. In: REUNIÃO ANUAL DA SOCIEDADE BRASILEIRA DE ZOOTECNIA, 33, Fortaleza, 1996. Anais... Fortaleza: SBZ, p.86-87, 1996.

PINHEIRO, M.G. Variação genética de características da capa externa de vacas da raça Holandesa em ambiente tropical. Ribeirão Preto: USP. 1996, 60p. Tese (Doutorado em Genética) - Universidade de São Paulo, 1996.

REIS, R.B. Fatores de variação da produção de leite, produção e porcentagem de gordura e período de lactação de vacas de diferentes graus de sangue Holandês. Belo Horizonte: 1983. 74p. Dissertação (Mestrado em Zootecnia) - Universidade Federal de Minas Gerais, 1983.

RIBAS, N.P., RORATO, P.R.N., LÔBO, R.B. et al. 1993. Estimativas de parâmetros genéticos para as características de produção da raça Holandesa no Estado do Paraná. R. Soc. Bras. Zootec., 22(4):634-641.

RITCHER, G.O., RIBAS, N.P., MONARDES, H.G. et al. Estudo da produção de leite, gordura e percentagem de gordura em vacas da raça Holandesa, região de Witmarsum, Paraná. In: REUNIÃO ANUAL DA SOCIEDADE BRASILEIRA DE ZOOTECNIA, 32, Brasília, 1995. Anais...Viçosa: SBZ, p.701-703, 1995.

RIVEROS MEDINA, A.A. Causas de variação e covariação de produçäo de leite. Belo Horizonte: UFMG, 1979. 86p. Dissertação (Mestrado em Zotecnia) - Universidade Federal de Minas Gerais, 1979.

RORATO, P.R.N. Interação genótipo-ambiente no desempenho produtivo da vacas da raça Holandesa. Ribeirão Preto: USP, 1988. 71p. Tese (Doutorado em Genética) -Universidade de São Paulo, 1988.

SILVA, M.V.G.B., BERGMAN, J.A.G., MARTINEZ, M.L. et al. Utilização de modelos animais uni e bivariados no estudo da eficiencia produtiva e reprodutiva na raça Holandesa. In: REUNIÃO ANUAL DA SOCIEDADE BRASILEIRA DE ZOOTECNIA, 32, Brasília, 1995. Anais... Viçosa: SBZ, p.637-639, 1995.

SPAIN, J.N. 1994. Why Missori dairy farms descontinue dairy herd improvement association testing programs. J. Dairy Sci., 77(4):1141-1145.

TEIXEIRA, N.M., FREITAS, A.F., VALENTE, J. et al. 1994. Tendências genéticas em rebanhos da raça Holandesa no Estado do Paraná II. Idade ao primeiro parto e primeiro intervalo de partos. R. Soc. Bras. Zootec., 23(6):992-1001.

TOMASZEWSKI, M.A. 1993. Record-keeping systems and control of data flow and information retrieval to manage harge high producing herds. J. Dairy Sci., 76(10):3188-3194.

VAN ARENDONK, J.A.M., HOVENIER, R., DE BOER, W. 1989. Phenotypic and genetic association between fertility and production in dairy cows. Lvstck. Prod. Sci., 21(1):1-12.

WIGGANS, G.R. 1994. Meeting the needs at the national level for genetic evalution an health monitoring. Symposium: meeting the information needs of the dairy industry. J. Dairy Sci., 77:1976-1983.
Recebido em: 12/05/98

Aceito em: 04/06/99 